\title{
Idiopathic true aneurysm of distal radial artery: case report
}

This article was published in the following Dove Press journal: Vascular Health and Risk Management

\author{
Nabil A Al-Zoubi \\ Department of Surgery, Jordan \\ University of Science and Technology, \\ Irbid, Jordan
}

Introduction: The incidence rate of true non-traumatic radial artery aneurysms (RAAs) is low, and very few cases have been described. The majority are traumatic in origin and are iatrogenic pseudo-aneurysms following arterial cannulation. However, other rare causes such as vascular tumors, connective tissue diseases, and occupational injury have also been reported. Only eight cases were reported as idiopathic true RAA. Herein, I describe a case of true idiopathic distal RAA, which was managed by surgical repair.

Case presentation: A 61-year-old-man with no significant medical history presented to vascular clinic with complaint of a nontraumatic mass on the volar aspect and radial side of his right wrist for 6 years. Duplex ultrasound and computed tomography angiogram revealed distal RAA. It was managed by open surgical resection with primary end-to-end anastomosis. Histopathological studies revealed true aneurysm of the distal radial artery.

Conclusion: True distal RAAs are an extremely rare entity. Unawareness of this condition might lead to inappropriate management with significant morbidity. Surgical management is generally indicated, but the best treatment remains controversial.

Keywords: radial artery, true aneurysm, idiopathic, arterial repair

\section{Introduction}

The upper limbs are considered less vulnerable to arterial diseases, especially those of aneurysmal nature, and radial artery is the least artery affected by aneurysmal disease after subclavian, brachial, and ulnar arteries. ${ }^{1}$ Radial artery aneurysms (RAAs) are rare entities comprising only $2.9 \%$ of all upper extremity aneurysms. ${ }^{2}$ They are mostly pseudoaneurysms and associated with trauma, usually penetrating or iatrogenic. ${ }^{1,3}$ However, true aneurysms of the radial artery are extremely rare. Only eight cases were reported as idiopathic true RAA. Herein, I report a case of an idiopathic isolated aneurysm in the distal radial artery with the clinical presentation, diagnostic modalities, and surgical management.

\section{Case presentation}

A 61-year-old male patient with insignificant medical history presented to vascular clinic from orthopedic clinic with complaint of a mass on the volar aspect and radial side of his right wrist (Figure 1). He started to notice this mass since 6 years. It was small and had gradually increased in size. He denied any history of trauma or previous cannulation at the same site. He is a barber and a nonsmoker. On physical examination, the mass was $3 \times 3 \mathrm{~cm}$, there were no skin changes, and it was pulsating. Allen's test
Correspondence: Nabil A Al-Zoubi

Department of Surgery, Jordan University of Science and Technology, Irbid 22I I0, Jordan

Tel +962 795774637

Email dr_nabeil_z3bi@yahoo.com 
was positive. There was no evidence of other aneurysms in both upper and lower limbs by physical examination. A trial of needle puncture of the mass was performed at orthopedic clinic before referral and revealed arterial blood flow, and so he was referred to our vascular clinic.

Doppler ultrasound and computed tomography (CT) angiogram (CTA) showed a $3 \times 3 \mathrm{~cm}$ isolated distal RAA with normal ulnar artery and intact both deep and superficial palmar arches (Figure 2).

On patient request and taking into account the potential risks such as thromboembolic complications or even rupture, a surgical repair was decided and performed. Under local anesthesia, a $4 \mathrm{~cm}$ longitudinal incision above the aneurysm was done. A saccular aneurysm was identified at the distal part of the radial artery after the takeoff of the superficial palmar arch branch (Figure 3). Then, the proximal, the distal radial artery, and the origin of the superficial palmar arch were controlled. Excision of the aneurysm was done, and the excised specimen was sent for histopathology. Arterial continuity was restored by primary end-to-end anastomosis between proximal and distal radial artery (Figure 4). The postoperative course was uneventful, and patient was discharged on the same day on simple analgesia. The histopathology report showed a dilated arterial sac with intraluminal mural thrombus.

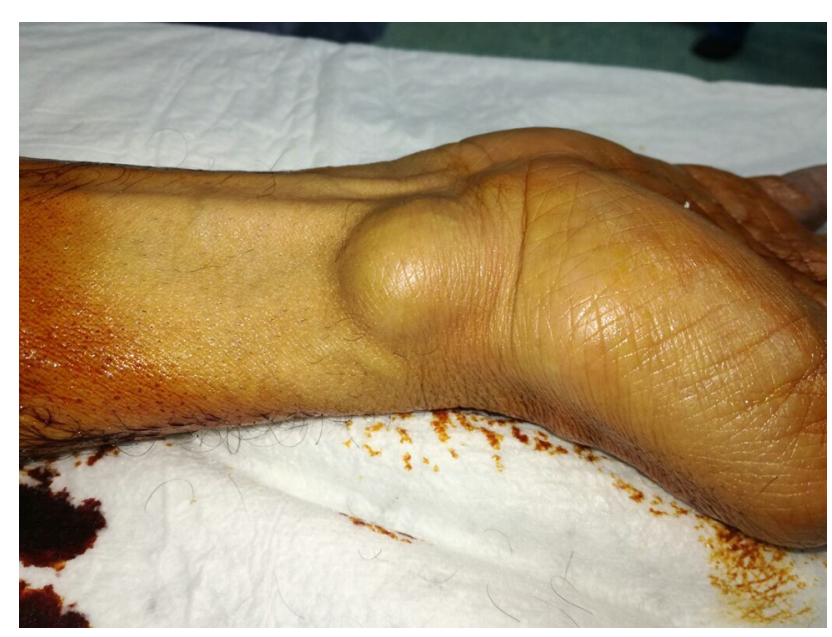

Figure I Right wrist swelling.

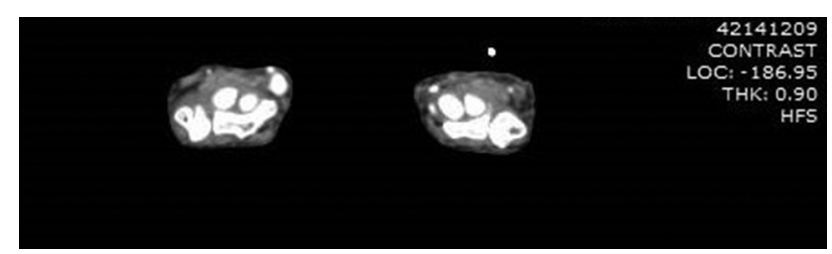

Figure 2 CTA of the distal radial artery aneurysm. Abbreviation: CTA, computed tomography angiogram.

\section{Discussion}

Shaabi et al reported that in 1966, RAA was first recognized by Thorrens, and since then some reports have been published. ${ }^{4}$ The majority of studies reported traumatic pseudoaneurysms, and only few authors reported cases of "true idiopathic" aneurysms. ${ }^{4}$

The initial diagnosis can be made clinically. ${ }^{5}$ Most cases present as an asymptomatic pulsatile swelling. ${ }^{6}$ It can be

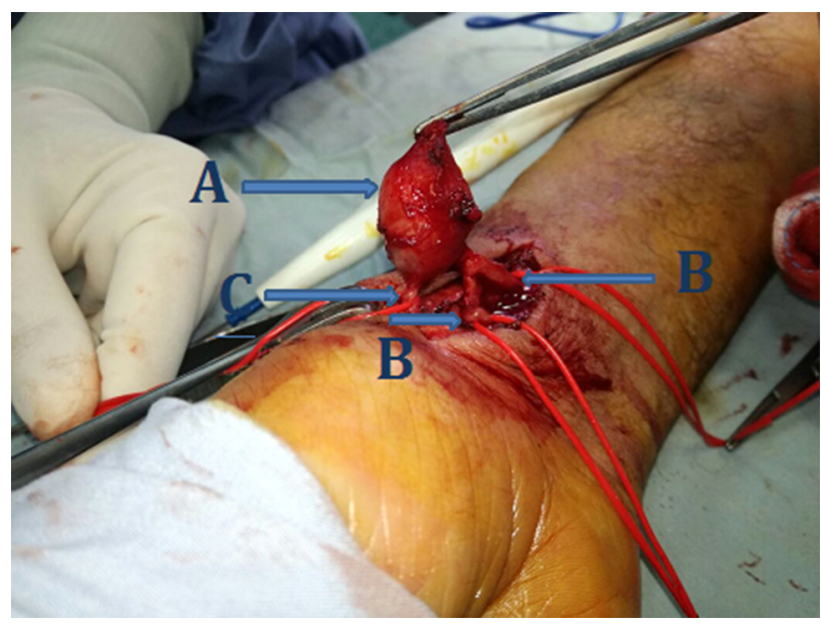

Figure 3 Intraoperative image showing A - aneurysm, B - radial artery, and C proximal part of the superficial palmar arch.

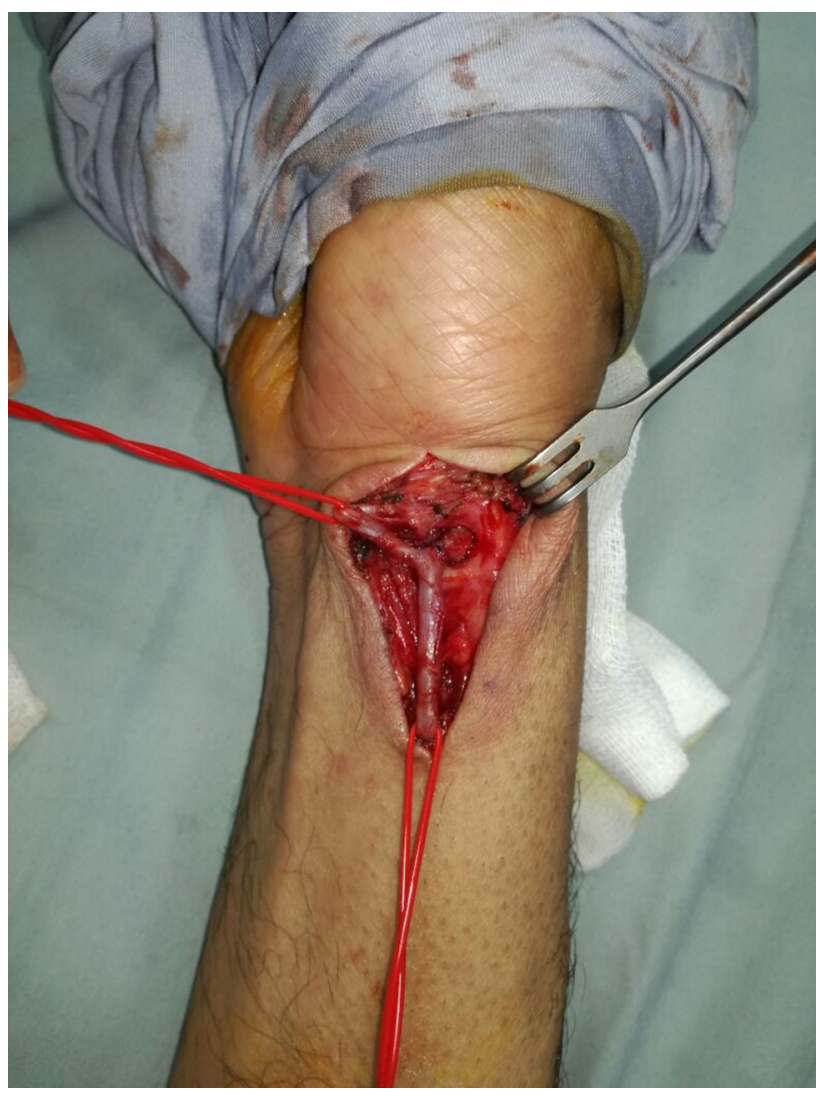

Figure 4 End-to-end anastomosis of the distal radial artery. 
presented with complications such as distal thromboembolism, digital ischemia, rupture, hemarthrosis, adjacent nerve irritation, paresthesia, limited wrist mobility, skin ulceration, and secondary infection. ${ }^{2}$ Misdiagnosis of a ganglion remains the pitfall. ${ }^{3}$ Other differential diagnosis includes synovial cyst, lipomas, and neuromas. ${ }^{1}$

Preoperative imaging will help in the diagnosis and to determine the most appropriate surgical management, including the need for vascular reconstruction. ${ }^{4,7}$ Duplex ultrasonography can play an important role in distinguishing true arterial aneurysms from pseudoaneurysms and the quality of in-line flow proximal and distal to the lesion in question. ${ }^{2}$ Preoperative angiography can also be a valuable tool to help identify associated vascular pathologies such as arteritides, arteriovenous fistulas and malformations, fibromuscular dysplasia, and venous anomalies. ${ }^{2}$

RAA should be treated rapidly because of the local and systemic risks of complications. ${ }^{2,8}$ The method of treatment remains controversial. ${ }^{1,3}$ It can include simple ligation, excision with primary anastomosis, or an interposition graft. ${ }^{6}$ Previous reports had concluded that the treatment of choice is resection of the aneurysm and arterial reconstruction with primary end-to-end anastomosis or proximal and distal ligature of the vessel. ${ }^{8}$ Others had reported that the most common approach for RAAs is ligation and excision, which appears to be the preferred in cases where the ulnar artery is the dominant arterial supply for the hand. ${ }^{6}$ Transcatheter therapeutic options such as covered stent exclusion, coil, and polymerizing agent embolization are extremely limited and ill advised given the local anatomical restrictions. ${ }^{2}$ In the case described here, although there was adequate reflux from the distal radial artery, the decision to restore arterial blood supply by end-to-end anastomosis of the radial artery was done because there was no segmental defect and healthy appearing arterial stumps.

\section{Conclusion}

Distal radial artery true aneurysms are extremely rare entity. Unawareness of this condition might lead to inappropriate management with significant morbidity. Surgical management is generally indicated, but treatment of choice remains controversial.

\section{Informed consent}

The patient gave a written informed consent for the case report and included images to be published.

\section{Disclosure}

The author reports no conflicts of interest in this work.

\section{References}

1. Meira LE, Gouvêa TM, Macedo Tjde. Idiopathic radial artery aneurysm: case report. J Vasc Bras. 2011;10(4):315-318.

2. Nassiri N, Kogan S, Truong H, Nagarsheth KJ, Shafritz R, Rahimi S. Surgical Repair of a Snuffbox Radial Artery Pseudoaneurysm. Clin Surg. 2016;1:1154

3. Walton NP, Choudhary F. Idiopathic radial artery aneurysm in the anatomical snuff box. Acta Orthop Belg. 2002;68(3):292-294.

4. Shaabi HI. True idiopathic saccular aneurysm of the radial artery. $J$ Surg Case Rep. 2014;2014(6):rju058.

5. Deşer SB, Demirağ MK. True Radial Artery Aneurysm Presenting With Behçet's Disease. Arch Rheumatol. 2017;32(2):177-178.

6. Thai H, Vasyluk A, Rits Y. Bilateral Radial Artery Aneurysm: Case Report and Review of Literature. Vasc Med Surg. 2016;4:247.

7. Jedynak J, Frydman G. Idiopathic True Aneurysm of the Radial Artery: A Rare Entity. Eur J Vasc Endovasc Surg. 2012;44(5):527.

8. Gabriel SA, De Abreu MF, De Abreu GC, et al. True posttraumatic radial artery aneurysm. J Vasc Bras. 2013;12(4):320-323.
Vascular Health and Risk Management

\section{Publish your work in this journal}

Vascular Health and Risk Management is an international, peerreviewed journal of therapeutics and risk management, focusing on concise rapid reporting of clinical studies on the processes involved in the maintenance of vascular health; the monitoring, prevention and treatment of vascular disease and its sequelae; and the involvement of

\section{Dovepress}

metabolic disorders, particularly diabetes. This journal is indexed on PubMed Central and MedLine. The manuscript management system is completely online and includes a very quick and fair peer-review system, which is all easy to use. Visit http://www.dovepress.com/ testimonials.php to read real quotes from published authors. 